\title{
In Search of a New Quantum Theory: From an Electron with a Volume to the Mechanism of Light Generation
}

\author{
Andrei Nechayev
}

\section{ABSTRACT}

\begin{abstract}
A new theoretical concept of quantum mechanics is proposed. The leading role is assigned to the electron as a non-point particle with a volume density of charge and mass. Based on the Hamilton-Jacobi equation, a nonlinear differential equation describing the dynamics of the charged substance of an electron is proposed. This new fundamental equation is transformed into the Schrödinger equation, with the density of the material substance of the electron being proportional to the square of the wave function. Since an electron in the form of a "cloud" of matter can change its configuration in space, we can give a classical interpretation to the process of generating a photon with a frequency and energy corresponding to the principles of quantum mechanics. Interference, diffraction, and the non-relativistic concept of electron spin is discussed.
\end{abstract}

Keywords: Axiomatic of quantum mechanics, photon generation, wave function, spin.
Published Online: April 13, 2021

ISSN: $2684-4451$

DOI :10.24018/ejphysics.2021.3.2.62

Andrei Nechayev*

Geographic Department, Moscow State

University, Russian Federation.

(e-mail: and.nechayev@gmail.com)

*Corresponding Author

\section{INTRODUCTION}

More than a hundred years have passed since Albert Einstein and Niels Bohr, convinced of the inapplicability of the laws of classical physics in the field of the microcosm and inspired by the discovery of Max Planck, proposed a new approach to the analysis of processes at the atomic level. A little less than a hundred years ago, the first works of Louis de Broglie, Erwin Schrodinger, and Werner Geiselberg were published, paving the way for understanding the "wave" properties of elementary particles and establishing quantum mechanics. In the future, many experiments, deeper and more refined, not refuted the foundations theory, exacerbated the questions of fundamental order. To what extent does the quantum mechanical description of nature correspond to reality? What is the reality that we observe? And do we need to try to explain and understand what in reality we still cannot observe?

In theoretical terms, the stationary Schrödinger equation, which made it possible to calculate the experimentally observed spectrum of the energy levels of the hydrogen atom, is indisputably reliable [1], [2]. However, the physical meaning of both the equation and its wave function is still undeciphered. Schrodinger himself tried to find a material basis for the wave function. For example, he wanted to represent a hydrogen atom as a nucleus, enveloped by an electron in the form of a charged cloud [3]. David Bohm made an important step towards classical theory that inspired many scientists. He found in the Schrodinger equation "roots" leading to the continuity and HamiltonJacobi equations [4]. However, his interpretation of the phenomenon continued to contain "virtual" parameters: the phase of the hypothetical De Broglie wave and the square of its amplitude as the probability density of measuring the particle coordinate.

This publication continues the movement begun in [5]-[7] along the "bridge" connecting quantum mechanics with classical mechanics.

\section{ACTION AND MATTER: THEIR RELATIONSHIP AND \\ BALANCE AT THE ATOMIC LEVEL. A NON-RELATIVISTIC EQUATION OF STATE FOR A MATERIAL SUBSTANCE}

So, using the Schrodinger equation as a "guiding star", we will begin our movement in a new direction in quantum theory. First of all, we will introduce the concept of matter as the primary liquid substance that makes up all the elementary particles of the universe (here and hereafter we will simply mean an electron by an elementary particle). This material substance has a density $\wp$, and the fact of the existence of an elementary particle is reduced to the following condition:

$$
\int \wp d V=1
$$

where $V$ is the volume of the particle. That is, (1) normalizes the amount of matter in one elementary particle. It is equal to one. Condition (1) formally follows from the assumption that the mass $m$ of the particle and its charge $e$ are distributed over the volume $V$ with densities $m \wp$ and $e \wp$. Matter, as a kind of liquid, can be in motion at a speed of $v$. We also assume that an elementary particle, in addition to mass and charge, has another immanent constant, namely, 
the quantum of action equal to $(-h / 2)$, where $h$ is Planck's constant. The immanent action is also distributed over the volume of the particle with the corresponding density $(-h / 2) \wp$. In the future, it will become clear that it is essential for us to use Planck's constant in its original form as a coefficient of proportionality between the energy of a light quantum and its frequency.

We regard action as one of the most fundamental characteristics of matter, although we find it difficult to explain its physical meaning. Initially, the action was a kind of mathematical abstraction, which was born thanks to the works of Maupertuis in the middle of the XVIII century. But this value was materialized only at the beginning of the $\mathrm{XX}$ century, after Planck discovered a constant $h$ that formed the foundation of a new theory - quantum mechanics. Action is inextricably linked to the energy content of matter, both at the macro and micro level. The total energy $E$ of a particle is determined by the total action $S$ in accordance with the Hamilton-Jacobi equation: $\partial S / \partial t+H=0$, where $H$ is equal to the sum of the kinetic and potential energy, that is, the total energy $E$. It follows that $S=-E t$. We assume that energy and action are distributed over a material substance with densities $E \wp$ and $-E \wp t$, so that the integral over the volume of the particle gives their full values. Thus, the density of the distributed action $S$ can be represented as the sum of the "external" action ( $-E \wp t)$ coming from outside, and the internal, "immanent" action $(-h / 2) \wp$. Since the change in action also occurs due to the divergence of internal flows determined by the factor $(-h / 2) \operatorname{div} \wp v$, we can form an analog of the Hamilton-Jacobi equation for a material substance in the form of an action transfer equation inside an elementary particle:

$$
-\frac{h}{2} \frac{\partial \wp}{\partial t}-\frac{h}{2} \operatorname{div} \wp \vec{v}-E \wp+\frac{m \wp v^{2}}{2}+U \wp=0
$$

where the kinetic and potential energy (the last two terms) are written in their classical sense (for example, in the case of an electron in a hydrogen atom, there is a Coulomb potential $U$ proportional to $e$ ).

Note that equation (2) can "break up" into two classical equations - continuity and conservation of energy provided that one of them is satisfied. In general, equation (2) combines the principles of conservation of mass and energy inside an elementary particle while providing the normalization condition (1).

To solve equation (2), it is necessary to determine the speed value $v$. To do this, we postulate an important condition that determines the dynamics of matter flows inside an elementary particle. It relates the density gradient of matter to the mass flow by analogy with the classical Fick's law (or Fourier's law), which establishes the proportionality between the concentration (or temperature) gradient and the corresponding flow. We will write this supposed law in canonical form: the gradient of the immanent action is equal to the density flow (internal momentum):

$$
-\frac{h}{2} \vec{\nabla} \wp=m \wp \vec{v}
$$

Postulate (3) imposes obvious limitations on the particle size. For example, an electron (more precisely, its "cloud") cannot "fall" on a proton, since the gradient of the substance $\nabla \wp$ in this case will tend to infinity, which will cause, in accordance with (3), a sharp increase in the rate of "expansion" of the electron cloud. Condition (3) essentially contains the Heisenberg uncertainty principle, being a physical basis for it. Indeed, the localization of a particle (for example, in the process of measuring its parameters) means an increase in the gradient modulus $\nabla \wp$, which leads to a corresponding increase in the internal momentum. We can estimate the order of the quantities included in (3). Introducing a certain average density $\bar{\wp}$ and average particle size $\Delta x$ (for the one-dimensional case), we rewrite (3) in the form $\frac{h}{2} \frac{\bar{\wp}}{\Delta x} \approx m \bar{\wp} v$ that gives the uncertainty relation: $m v \Delta x \approx \frac{h}{2}$.

Now we use condition (3) to simplify equation (2) and write it in the final form available for analysis:

$$
-\frac{h}{2} \frac{\partial \wp}{\partial t}-\frac{h^{2}}{4 m} \nabla^{2} \wp-E \wp+\frac{h^{2}}{8 m}(\nabla \wp)^{2}+U \wp=0
$$

Equation (4) can be considered as a kind of action transfer equation, where there is a "diffusion" term (the second one in the equation), where the total energy $E \wp$ acts as the "source" of the action, and the potential energy $U \wp$ acts as the "drain". In this case, there is a nonlinear term associated with the kinetic energy of internal flows, proportional to the square of the density gradient. As we will see later, this is an equation that can be called AMT (Action-Matter-Transfer)an equation that determines the state of an elementary particle. This is easily confirmed by a simple substitution $\wp=\psi^{2}$ that transforms equation (4) into the classical Schrodinger equation. Indeed, for the second and fourth terms of the equation, we have:

$$
\begin{aligned}
& \nabla^{2} \wp=\nabla(2 \psi \nabla \psi)=2(\nabla \psi)^{2}+2 \psi \nabla^{2} \psi \\
& (\nabla \wp)^{2}=(2 \psi \nabla \psi)^{2}=4 \psi^{2}(\nabla \psi)^{2}
\end{aligned}
$$

Substituting (5) in (4) and making the corresponding reductions, we get:

$$
-h \frac{\partial \psi}{\partial t}=\frac{h^{2}}{2 m} \Delta^{2} \psi+\psi(E-U)
$$

And no imaginary unit! At the same time, for the transition to the stationary case, it is not necessary to assume a hypothetical wave propagating in an unknown direction but having a de Broglie wavelength. It is enough to "stop" a certain transition process going on inside the particle (we 
will talk about it later), and get a stationary AMT equation from (4):

$$
-\frac{h^{2}}{4 m} \nabla^{2} \wp+\frac{h^{2}}{8 m}(\nabla \wp)^{2}=E \wp-U \wp
$$

which is transformed by substitution $\wp=\psi^{2}$ into the corresponding stationary Schrodinger equation. Given the above, it can be assumed that the Schrodinger equation and the wave functions should be considered as auxiliary mathematical attributes necessary for finding solutions to the stationary AMT equation (7), but possibly having no physical meaning.

\section{THE PRINCIPLE OF SUPERPOSITION. INTERFERENCE AND DIFFRACTION OF ELECTRONS}

The well-known quantum-mechanical superposition principle is based on the linearity of the Schrodinger equation, which allows solutions in the form of a sum (or linear combination) of eigenfunctions. This conclusion is trivial for degenerate states that have the same energy value $E$, and is not at all obvious for states with different energies. However, in the case of the AMT equation, the superposition principle does not work in principle, since this equation is essentially nonlinear and the sum of the states $\wp_{1}$ and $\wp_{2}$ it is not the decision. However, using the genetic connection of the AMT equation with the Schrodinger equation and the fact that any solution $\psi$ of the Schrodinger equation uniquely corresponds to the solution $\wp=\psi^{2}$ of the AMT equation, we can get a simple interpretation of the placement of electrons in atomic orbitals. Indeed, any sum of wave functions $N$ that are degenerate in energy satisfies the Schrodinger equation. Therefore, the AMT equation is satisfied by a function of the form:

$$
\wp=\left(\sum_{i=1}^{N} \psi_{i}\right)^{2}
$$

It is clear that state (8) satisfies the condition of normalizing the amount of matter $\int \wp d V=N$, since the integrals of all paired products of wave functions of the form $\psi_{i} \psi_{j}(i \neq j)$ are zero due to the orthogonality of these functions. And we get a state of $N$ electrons with a density distributed as follows:

$$
\wp=\sum_{i=1}^{N} \wp_{i}+\sum_{i, j=1}^{N} 2 \psi_{i} \psi_{j}
$$

where in the first term all states in the one-electron approximation are summed, and in the second term all except $(i=j)$.

Thus, each electron has its own function $\wp_{i}=\psi_{i}^{2}$ that characterizes the distribution of the density of its matter in space. Everyone $\wp_{i}$ is different. Which is provided by a different set of quantum numbers $n, l, m$ (the Pauli principle). The conservation of mass and the indivisibility of electrons guarantees the normalization condition $\int \wp d V=N$. In this case, the formula (8) implies the overlap of material substances. How this can happen is one of the many questions raised by the new theoretical concept presented in this paper. Indeed, despite the overlap of substances and "interference", each electron, apparently, retains its "privacy" and at any time can separate, free itself, having received the necessary energy for this.

How do free electrons behave in a collimated beam? Obviously, the electron cloud is very well compressible and can occupy the smallest volumes both outside and inside the atom. What happens at the boundary of two electronic substances? It is possible that there is a condition for the equality of the opposite gradients of matter $\nabla \wp$ to compensate for the mutual flows of substance caused by condition (3). One thing is clear: the AMT equation (7) can adapt each electron as a region of distributed charge eøo and include the corresponding potential in the function $U$, but this will greatly complicate the analysis and calculations.

Before analyzing the circumstances of the well-known experiment on the interference of electrons passing through two parallel slits, we will discuss how, based on our ideas about the structure of the electron, to interpret the registration of particles, that is, the measurement of their position in space.

Registration of fast particles, in particular electrons, is carried out using methods that use the kinetic energy of the particle. Thus, during scintillation registration, the energy of the collision causes flashes of light - the emission of photons. And when registered by the method of thick-layer photoemulsion, the collision of particles with a film or plate causes ionization of bromine atoms and the corresponding blackening. In the Wilson chamber, there is a collision with water vapor. In any case, following our ideas, we can assume that a particle having a distributed density $m \wp$ and moving at a speed $v$, it interacts with the registering object in proportion to its local kinetic energy $m \wp v^{2} / 2$, which also has a distribution in space. That is, where the density of matter is higher, the impact on the recording surface will be stronger and there will be a greater probability of the corresponding fixation of the particle position.

Now let's turn to the experiment with two slits. In its various variants, a beam of electrons accelerated by a potential difference $U$ to energy $e U$ was separated into two streams using slits or a thin wire and then hit the screen, where the electron registration gave an interference pattern. The interpretation of the observed effect based on the AMT equation does not formally differ from the "classical" quantum-mechanical interpretation. Indeed, two electrons, each passing through its own slit, represent "clouds" of matter with modulated density. The nearest pair of electrons makes contact. Their general state should be described by a function $\wp_{12}$ that satisfies the AMT equation and the normalization condition $\int \wp_{12} d V=2$. Since both electrons have the same energy $E=e U$, their states $\wp_{1}$ and $\wp_{2}$ 
correspond to sinusoidal wave functions $\psi_{1}$ and $\psi_{2}$ with the same de Broglie wavelength $h / \sqrt{2 m E}$. These functions satisfy the Schrodinger equation for a free electron, and it can be assumed that the desired state $\wp_{12}$ of two electrons passing through the gap should be described as follows:

$$
\begin{aligned}
& -\frac{h}{2} \frac{\partial f}{\partial t}-\Delta E f=0 \\
& -E F-\frac{h^{2}}{4 m} \nabla^{2} F+\frac{h^{2}}{8 m}(\nabla F)^{2}+U F=0
\end{aligned}
$$

$$
\wp_{12}=A\left(\psi_{1}+\psi_{2}\right)^{2}=A\left(\psi_{1}^{2}+\psi_{2}^{2}+2 \psi_{1} \psi_{2}\right)=A\left(\wp_{1}+\wp_{2}+2 \psi_{1} \psi_{2}\right) \text { The second equation from (11) is the stationary AMT }
$$

The relevant question is: when and how does an electron "learn" about its kinetic energy? When does it accelerate or when does it slow down? Because during a uniform motion, due to the principle of relativity, it must be "insensitive". And there should be no waves in it, neither standing nor running! I would like to think that the energy received by the electron during acceleration by the field enters it in collisions: with the anode, with the collimator, with two slits. And it is then that all the processes take place in it, because the AMT equation begins to work.

\section{THE EMISSION OF A PHOTON}

The absorption and emission of a photon is a quantum process. But in order to use the classical Maxwell equations for its interpretation, we need to find charges that change in space and time. To do this, we use the non-stationary AMT equation (4). It describes the redistribution of the density of matter, and hence the mass and charge in the electronic "cloud". Suppose that an electron is in some stationary state $\wp_{0}$ with energy $E_{0}$ and energy $\Delta E>0$ is transferred to it from the outside. The matter of the electron will be rearranged in accordance with the AMT equation and go from a state $\wp_{0}$, for example, to a state $\wp_{1}$ if $\Delta E=E_{1}-E_{0}$. The whole process will be described by the following equation:

$$
-\frac{h}{2} \frac{\partial \wp}{\partial t}-(E+\Delta E) \wp-\frac{h^{2}}{4 m} \nabla^{2} \wp+\frac{h^{2}}{8 m}(\nabla \wp)^{2}+U \wp=0
$$

During the transition of an electron from one state to another, the distribution of its charge eø changes: for example, after the transition of an atom to a higher energy level, the distributed charge of the electron is at a greater distance from the nucleus. The main quantum number of the hydrogen atom determines the average radius of the electron "cloud", during transitions from one energy level to another, the "cloud" pulsates: it contracts, approaching the positive core, then expands, moving away from it. This process is actually analogous to the oscillations of an electric dipole: a pulsating electronic "cloud" must emit an electromagnetic wave. The frequency of the pulsations and the length of the corresponding wave must correspond to the time of transition from one state to another. Let us try to estimate this time using equation (10). Let us represent the density of matter as the product of two functions: $\wp=f(t) F$, where $F$ depends only on the spatial coordinates. After substituting this representation into equation (10), it is split into two equations: $f=\exp (-t / \tau)$ with a characteristic time $\tau=h / 2 \Delta E$. We discussed the transition of an electron from a low level to a high level after the electron receives additional energy $\Delta E$. A symmetric process with the same characteristic time should be expected in the reverse transition from a high level to a low level if the energy is taken away. The electron "cloud" will then return to its previous state, completing its elementary oscillation, during which the photon should be emitted. The first half period of the wave is emitted when the "cloud" in the transition of the atom to a higher energy level, the second half-cycle is emitted during compression clouds when you return the atom to its previous level. The entire period is $2 \tau=h / \Delta E$. The inverse of this period gives the frequency $v$ of radiation, and if the" pulsations " of the electron cloud are repeated (and this is possible if energy is constantly supplied to the atom from outside, for example, as a result of heating), then we get the classical: $\Delta E=h v$.

The above description of the photon emission process has the advantage: it represents the photon as a particle of an electromagnetic wave, limited in space and time. Inside this particle-wave, the field strength should change the amplitude and sign, respectively, of the first and second half-periods of the pulsation of the electron "cloud", which serves as the basis for the interference of photons.

\section{SPIN: A NON-RELATIVISTIC INTERPRETATION}

The proper mechanical moment of rotation of the electron (spin) is said to be an established fact. Indeed, this moment and the accompanying magnetic moment allow us to explain many quantum mechanical effects.

In 1925, Uhlenbeck and Goudsmith proposed the spin hypothesis, suggesting that an electron is like a rotating charged ball and must have an immanent mechanical and magnetic moment. But soon they actually abandoned their idea [8], because the electron (if we take its radius of the order of $10^{-15} \mathrm{~m}$ ) will rotate at superluminal speed. However, if we follow our concept and consider the electron as a charged "cloud" with a size corresponding to the spatial dimensions of the wave function of the Schrodinger equation, then the contradiction with the theory of relativity can be removed. In our paper, we want to show that spin can have a classical interpretation.

Since electrons in our understanding are by no means point particles, but rather a rather vague substance of the lightest matter that forms the actual dimensions of atoms and molecules, we can leave aside all questions related to the theory of relativity and believe that the electron cloud may well rotate around one of its innumerable axes at speeds much lower than the speed of light. Moreover, the bulk of this "cloud" is concentrated at a distance no less than 
the radius of the Boron.

Since, from our point of view, an electron cannot have an orbital moment (an electron does not rotate around an atom in any plane as a point particle), and the quantum numbers $l$ and $m$ determine not the inclination of its orbit, but the configuration of a particular state of the electron cloud $\wp$ for a given energy level. But the proper rotation of the electron cloud is possible, and it not only explains the Stern-Gerlach experiment, causing the desired magnetic moment, but also facilitates the understanding of the possibility of the existence of stable electronic configurations in the shells of atoms with more than one proton.

First, we note that the spin of an electron in a "cloud" around the nucleus should not cause the emission of electromagnetic waves (in contrast to the point of rotation of the electron), because the spatial distribution of charge e£ during the rotation may not change if the axis of rotation perpendicular to the plane of symmetry passes through the core. This is most obvious for the spherical $s$-states of the electron, as well as for the dumbbell like $p$-and $d$-states.

We can verify that the gyromagnetic ratio of the rotating electron cloud is equal to $e / 2 m$, which formally corresponds to the orbital rotation of a point electron in the Bohr orbit. Indeed, we will represent this cloud in a cylindrical coordinate system and divide it along the axis of rotation $z$ into flat disks with a thickness of $d z$. The modulus of the mechanical moment $M$ of each such disk will be equal to:

$$
M=d z \int m \wp 2 \pi r v r d r=d z 2 m \pi \int \wp v r^{2} d r
$$

where $v$ is the azimuthal rotation speed of an elementary ring with a radius $r$, width $d r$, and mass density $m \wp$. The corresponding modulus of the magnetic moment of such a ring will be equal to:

$$
\mu=d z \int e \wp v \pi r^{2} d r=d z e \pi \int \wp v r^{2} d r
$$

where eøv is the current density of the charged matter that envelopes the circle with an area of $\pi r^{2}$.

The gyromagnetic ratio $\mu / M$ for these disks, as well as for the entire electron cloud, will obviously be equal $e / 2 \mathrm{~m}$. Note that we have considered a fairly general case of rotation, not limited only to" solid-state " rotation with a constant angular velocity.

Indeed, the rotation of the electron cloud around a certain axis can be represented as a set of rotating charged rings (a set of elementary ring currents), which must respond to an external magnetic field if it is directed perpendicular to the plane of rotation. So, each element $d l$ of this ring will be affected by an Ampere force proportional to øevdlB, where $B$ is the magnetic induction, $v$ is the speed of rotation of the ring. This force will always be directed along the radius of rotation according to the right-hand rule: to the center if the rotation is counterclockwise, and from the center if the rotation is clockwise. That is, depending on the direction of its rotation, the electron cloud in the magnetic field will either contract or stretch, and its energy will either increase (stretching) or decrease (compression). This gives us a doublet: splitting each energy level for an electron cloud oriented along the field.

Consider the supposed electronic configuration of the helium atom, whose nucleus has two protons. The outer shell of a helium atom contains two electrons in S-states (with different spins), similar to the ground state of the hydrogen atom, only with a different energy level and a different average distance from the nucleus. The density of matter (the square of the corresponding wave function) is a spherically symmetric function. Why can't each electron cloud occupy its own hemisphere (with doubled density of charge and mass)? Then two hemispheres of two electrons will occupy the entire space around the nucleus, and their densities will satisfy the AMT equation, if we accept certain boundary conditions in the contact zone of two electronic substances. It is also clear that the rotation of these hemispheres in one direction around the vertical axis, firstly, does not change the charge distribution (and the atom does not radiate), and secondly, it turns them into elementary magnets that are attracted to each other. The mutual magnetic attraction of these unidirectionally rotating hemispheres provides the helium atom with additional stability and inertia.

\section{CONCLUSIONS}

A new approach to the axiomatic of quantum mechanics is proposed. The physical basis of this approach is the rejection of the representation of the electron as a point particle. For the dynamic description of the material substance of the electron, an equation is proposed that is genetically related to the Hamilton-Jacobi equation. By a simple transformation, this equation becomes the Schrodinger equation, and the dimensionless density of the material substance of the electron is equal to the square of the wave function. The presence of a material substance in an electron allows us to interpret the light emission as the generation of a chain of electromagnetic pulses as a result of the pulsation of the volume of this charged substance. And its three-dimensional rotation is a logical explanation for spin.

\section{REFERENCES}

[1] Schrödinger E., Ann. Phys., 1926, v.384, p.361

[2] Schrödinger E., Phys. Rev., 1926, v.28, p.1049.

[3] Four Lectures on Wave Mechanics... by Dr. Erwin Schrodinger. Blackie \& Son Limited, London and Glasgow, 1928

[4] Bohm D., Phys. Rev., 1952, v.85, p.166.

[5] Nechayev A. On the fundamental properties of matter and action: A new quasiclassical concept of quantum mechanics, Physics Essays, 2015, v.28, n3. p.331-333.

[6] Nechayev A. A New Theoretical Concept of Quantum Mechanics and a Quasi-Classical Interpretation of the Fundamental QuantumMechanical Experiment. Chapter in the book "Quantum mechanics, Theory, Analysis and Applications" (Nova Science Publishers, New York, USA, 2018) ISBN: 978-1-53614-208-2.

[7] Andrei Nechayev, On the Semi-classical Approach to the Physical Axiomatic of Quantum Mechanics and the New Wave-Particle Interpretation of Light, American Journal of Modern Physics. Vol. 9 , No. 3, 2020, pp. 48-54

[8] Uhlenbeck G., Gaudsmit S., Nature, 1926, v.117, p.264-265. 